\title{
Erfolgreiche Behandlung einer nekrotisierenden, multiresistenten bakteriellen Pyodermie bei einem Python mittels Kaltplasmatherapie
}

\author{
Christoph J. Klinger ${ }^{1}$; Berrett Dengler ${ }^{1}$; Thomas Bauer²; Ralf S. Mueller ${ }^{1}$ \\ ${ }^{1}$ Medizinische Kleintierklinik, Zentrum für Tiermedizin, Ludwig-Maximilians-Universität, München; ${ }^{2}$ Tierarztpraxis für Reptilien und Exoten, München
}

\author{
Schlüsselwörter \\ Kaltplasma, Atmosphärendruckplasma, Dermatologie, Reptilien, Ste- \\ notrophomonas maltophilia, Xanthomonas
}

\section{Zusammenfassung}

Ein 4-jähriger Königspython wurde 3 Wochen nach Bissverletzungen durch eine Ratte mit großflächigen Hautläsionen und einer bakteriellen Pyodermie mit Anzeichen einer Sepsis und neurologischen Symptomen vorgestellt. Die betroffene Haut löste sich von der Muskulatur, wodurch Teile des Muskelgewebes frei lagen. Klinische und zytologische Untersuchung ergaben eine bakterielle Pyodermie. Sepsisverdacht bestand aufgrund der klinischen Symptomatik. Die empirische Behandlung mit Lincomycin und Marbofloxacin (Befund der bakteriologischen Untersuchung waren multiresistente Stenotrophomonas maltophilia, sensibel gegenüber Fluorchinolonen) verbesserte das Allgemeinbefinden und die Hautläsionen demarkierten sich zu Brandschorf mit intrazellulären stäbchenförmigen Bakterien. Eine weiterführende Diagnostik konnte kostenbedingt nicht durchgeführt werden und die Euthanasie des Tieres wurde erwogen. Im Verlauf von 4 Wochen erfolgte sechsmalig eine Behandlung mit Kaltplasma (cold atmospheric pressure plasma, CAPP). Innerhalb einer Woche verbesserten sich die Entzündungssymptome. Die Reepithelisierung war wenige Wochen später abgeschlossen. Drei Häutungen im Folgejahr verliefen ohne Anzeichen von Dysecdysis. CAPP könnte bei Schlangen mit bakteriellen Pyodermien (insbesondere mit resistenten Keimen) und Hautnekrosen eine nützliche Behandlungsoption darstellen.

\section{Keywords}

Non-thermal plasma, atmospheric pressure plasma, dermatology, reptiles, Stenotrophomonas maltophilia, Xanthomonas

Summary

A 4-year-old ball python was presented 3 weeks after multiple bite wounds from a prey rat with large skin lesions, a concurrent deep bacterial pyoderma and clinical signs for septicemia, including neurological symptoms. Affected tissue separated from the underlying muscular layer revealing parts of the muscles. Clinical examination and cytology was consistent with bacterial pyoderma; septicemia was an additional tentative clinical diagnosis. Empirical lincomycin and marbofloxacin (bacterial culture revealed a multi-resistant Stenotrophomonas maltophilia susceptible to fluoroquinolones) treatment improved the patient's general condition but skin wounds deteriorated to multifocal eschars with intracellular rods. Further diagnostics were limited for financial reasons, euthanasia was considered. Cold atmospheric pressure plasma (CAPP) therapy was performed six times in 4 weeks. Within 1 week, inflammatory symptoms resolved. Re-epithelialization was completed few weeks later. In the following year, the snake sloughed three times without any signs of dysecdysis. CAPP therapy may offer a viable treatment option for bacterial (especially multi-resistant) pyoderma and necrotizing dermatitis in snakes.

Successful treatment of a necrotizing, multi-resistant bacterial pyoderma in a python with cold plasma therapy

Tierarztl Prax Ausg K Kleintiere Heimtiere 2018; 46: 43-48

http://dx.doi.org/10.15654/TPK-170190

Eingegangen: 8. März 2017

Akzeptiert nach Revision: 26. Mai 2017

Veterinärstraße 13

80539 München

E-Mail: c.klinger@medizinische-kleintierklinik.de

\section{Einleitung}

Der Königspython (Python regius, SHAW 1802) ist eine ovipare, ungiftige Würgeschlangenspezies, die im subsaharischen Afrika beheimatet ist (36). Da Königspythons mit zu den kleinsten afrikanischen Riesenschlangen gehören und - solange sie nicht bedroht werden - relativ friedliebend sind, werden sie oft als Haustiere gehalten.

Bakterielle Infektion stellen bei Reptilien ein häufiges Problem dar (6) und werden oftmals durch gramnegative Stäbchen verursacht (31). Einige dieser Bakterien - ungeachtet des Trägers bergen ein hohes Potenzial, Resistenzen gegen Antibiotika zu ent- 
wickeln, selbst ohne vorangegangenen Einsatz derselbigen (29, 30). In vielen Fällen ist eine antibakterielle Therapie vonnöten, da topisch applizierte Medikamente oder desinfizierende Bäder schlecht toleriert werden, ineffektiv sind $(16,33)$ oder das sehr dicke, schützende Stratum corneum nicht penetrieren $(19,24)$. Einige Reptilienarten entwickeln jedoch sehr schnell Nebenwirkungen auf Antibiotika wie z. B. Nephrotoxizität (26). Daher muss manchmal auch die Euthanasie als Ultima Ratio in Betracht gezogen werden.

Stenotrophomonas maltophilia ist ein ubiquitäres, aerobes, gramnegatives, stäbchenförmiges Bakterium der Familie Xanthomonadaceae, das nachgewiesenermaßen schwere septikämische Infektionen bei Reptilien auslösen kann $(1,11)$ und oftmals Multiresistenz aufweist (6).

Bei Kaltplasma (cold atmospheric pressure plasma, CAPP) handelt es sich um eine relative neue, innovative Therapieoption mit physikalischer antimikrobieller Aktivität und dem Potenzial zur Wundheilungsförderung (37). Man kann es sich als eine gasförmige Mischung aus Elektronen und Ionen in Kombination mit neutralen und hochreaktiven Molekülen wie freien Radikalen, Peroxiden und UV-Photonen vorstellen $(9,20)$. CAPP inaktiviert Bakterien ungeachtet deren Resistenzen gegenüber antimikrobiellen Substanzen, wie z. B. den Methicillin-resistenten Staphylococcus aureus und Pseudomonas aeruginosa (2), und zerstört gleichzeitig bakterielle Biofilme, Bakteriophagen sowie Bakterien- und Pilzsporen $(18,35)$.

Dieser Fallbericht beschreibt einen mit multiresistentem Stenotrophomonas maltophilia infizierten Königspython, der mittels CAPP in Kombination mit lokaler Therapie behandelt wurde. Nach bestem Wissen der Autoren ist dies der erste Fallbericht über den Einsatz der Kaltplasmatherapie bei einem Reptil mit multiresistenter bakterieller Infektion.

\section{Fallbeschreibung}

\section{Vorbericht und klinische Präsentation}

Ein weiblicher, 1,5 m langer, 4 Jahre alter Königspython wurde aufgrund von Anorexie, Lethargie und neun großen sowie multiplen kleinen teils vollständig ulzerierten Hautstellen mit Punktionswunden am gesamten Körper vorgestellt. Der Besitzer berichtete, er setze regelmäßig lebende Ratten als Futterquelle in das Terrarium ein. Drei Wochen vor der Erstvorstellung bei einem Reptilienspezialisten beobachtete der Besitzer, wie eine Ratte dem Python multiple Bissverletzungen zufügte, als dieser auf sie zuschnellte. Die Schlange tötete daraufhin diese Ratte nicht und der Besitzer entfernte das Futtertier aus dem Terrarium. Auf Nachfrage versicherte der Besitzer, im Terrarium gebe es keinerlei Kontakt-Hitzequellen und keine scharfen Objekte, die die Hautwunden hätten verursachen können.

Die Schlange war untergewichtig, versuchte nicht, der Allgemeinuntersuchung zu entkommen, und wies einen deutlichen Opisthotonus auf. Bei Positionierung in Rückenlage zeigte sie kei- nerlei physiologische Kompensationsbewegungen. Im Ventrumbereich fielen zahlreiche punktuelle petechiale Blutungen auf. Die Größe der Wunden variierte von $1 \mathrm{~cm}^{2}$ bis hin zu $15 \times 7 \mathrm{~cm}$.

\section{Diagnostik, Behandlung und Therapieerfolg}

Die direkte zytologische Untersuchung von Proben aus dem Bereich kleiner Ulzerationen ergab heterophile Granulozyten und intrazelluläre, stäbchenförmige Bakterien im Einklang mit einer bakteriellen Pyodermie. Die Petechien und neurologischen Anzeichen deuteten auf eine Septikämie hin. Der Patient wurde stationär aufgenommen und Tupferproben von der freiliegenden Muskeloberfläche unterhalb der großen Hautwunden entnommen und zur bakteriologischen Untersuchung mit Antibiogramm an ein kommerzielles Labor versandt. Aufgrund finanzieller Limitationen verweigerte der Besitzer jegliche weiterführende Diagnostik.

Die empirische initiale Behandlung umfasste intramuskuläre Injektionen von Marbofloxacin (Marbocyl ${ }^{\circ} 2 \%$, Vetoquinol, Magny-Vernois, Frankreich) in einer Dosis von $4 \mathrm{mg} / \mathrm{kg} / \mathrm{d}$ (initial einmalig $8 \mathrm{mg} / \mathrm{kg}$ ) und Lincomycin (Lincobel ${ }^{\circ} 113,4 \mathrm{mg} / \mathrm{ml}$, belapharm GmbH \& Co. KG, Vechta) in einer Dosis von $10 \mathrm{mg} / \mathrm{kg} / \mathrm{d}$ in Kombination mit subkutanen Infusionen von physiologischer Vollelektrolytlösung (Ringer-Lactat-Lösung nach Hartmann", B. Braun Vet Care, Melsungen) in einer Dosis von $10 \mathrm{ml} / \mathrm{kg} / \mathrm{d}$. Begleitend dazu wurde topisch mit Silber-Sulfadiazin-Salbe (Flammazine ${ }^{\oplus} 1 \%$, Sinclair Pharma GmbH, Frankfurt/Main) zweimal täglich behandelt, nachdem die Schlange zuvor jeweils in hochkonzentriertem Schwarztee (gekühlt auf $25^{\circ} \mathrm{C}$ ) gebadet worden war. Die bakterielle Kultur ergab solitär, aber in hohen Mengen vorhandene Stenotrophomonas maltophilia, die resistent auf alle getesteten Antibiotika außer Fluorchinolone, Trimethoprim-Sulfonamide und Chloramphenicol/Florfenicol waren. Die systemische und die topische Therapie wurden folglich über eine Woche bei vorsichtiger Prognose fortgesetzt.

Zehn Tage später wies der Patient ein deutlich verbessertes Allgemeinbefinden auf und der Python war nun in der Lage, seine Position aus der Rückenlage heraus selbständig zu korrigieren. Der Zustand der Hautwunden hatte sich jedoch zunehmend verschlechtert. Multifokal fand sich Brandschorf über die gesamte Dicke der Epidermis, nekrotisierte Haut löste sich ab mit Freilegung der darunterliegenden Muskulatur ( $\triangleright$ Abb. 1). Der Patient wurde für eine Alternative zur Euthanasie an die Abteilung für Dermatologie überwiesen. Eine erneute zytologische Untersuchung der Muskeloberfläche zeigte große Mengen an Heterophilen und intrazellulären Stäbchen. Einer weiterführenden Diagnostik stimmte der Besitzer nicht zu. Daraufhin wurde eine Behandlung mit Kaltplasma (kINPen VET'; neoplas GmbH, Greifswald) zur Desinfektion und Induktion der Wundheilung vorgeschlagen. Diese erfolgte über 2 Wochen dreimal wöchentlich für $15 \mathrm{~s} / \mathrm{cm}^{2}$ mit einem Jet-Wunden-Abstand von $10 \mathrm{~mm}(\triangleright \mathrm{Abb} .2)$. Die topische Applikation von Silber-Sulfadiazin fand als unterstützende Therapie zur CAPP-Behandlung weiterhin statt; die Teebäder und die systemische Antibiose wurden abgesetzt. 


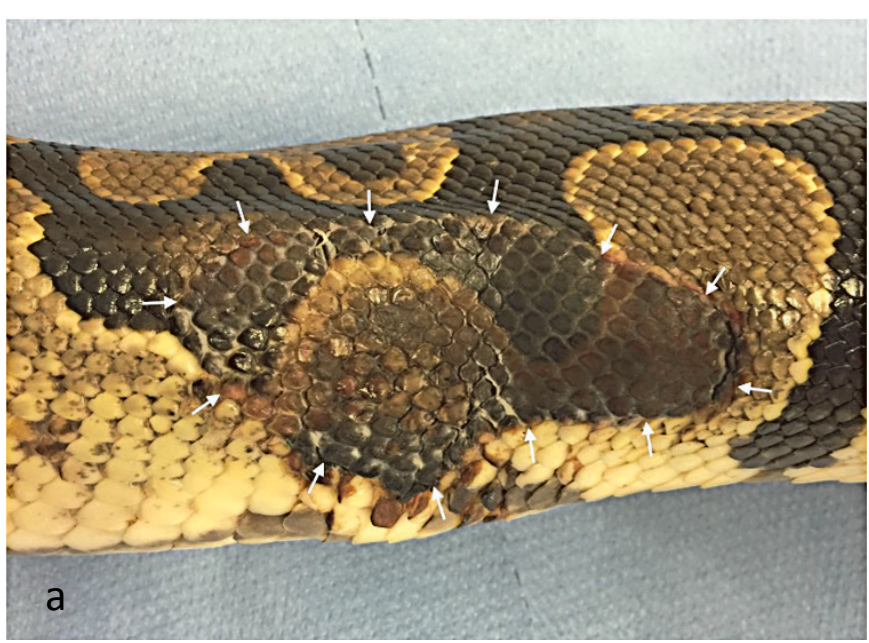

Abb. 1 Klinisches Bild des Königspythons. a) Scharf demarkiertes, nekrotisierendes Hautareal an der rechten Flanke im mittleren Körpersegment. b) Sich teilweise ablösender Brandschorf an der Brustwand mit Freilegung der darunterliegenden Muskulatur.

Nach 7 Tagen war Granulationsgewebe im Wundrandbereich sichtbar und bei der zytologischen Untersuchung fanden sich keine Bakterien mehr. Nach 14 Tagen waren die nekrotischen Areale in ihrer Fläche um etwa 50\% reduziert, ohne dass weitere Stellen mit Gewebeverlust auftraten. Die CAPP-Therapie wurde nach weiteren 2 Wochen mit jeweils einmaliger Anwendung beendet. Zwei Monate später waren alle Hautwunden komplett zugeheilt und hinterließen lediglich deutliches Narbengewebe, das im weiteren Verlauf von 12 Monaten zu keinen Häutungsproblemen führte ( Abb. 3).

\section{Diskussion}

Die Lebendfütterung von Reptilien beinhaltet einige Risiken, da sich Beutetiere oft durch Bisse oder Kratzer gegen ihre Angreifer verteidigen (4). Sie ist ein kontrovers diskutiertes Thema unter Experten, nicht nur wegen potenzieller Verletzungen der Schlange (4), sondern auch aufgrund ethischer Bedenken bezüglich der Beutetiere (Art. 4 Abs. 3 TSchV). Daher wurde dem Besitzer zu einer Umstellung der Fütterung auf frisch getötete oder aufgetaute gefrorene Beutetiere geraten.

Der Tierhalter hatte die Bissverletzungen durch die Beuteratte direkt beobachtet. Bei der Erstvorstellung der Schlange bei einem Reptilienspezialisten schätzte dieser die vorliegenden Wunden passend zu diesem Ereignis als tiefe Punktionswunden ein.

Königspythons zeigen in Gefangenschaft unter Stress häufiger Phasen von Anorexie und können über Monate hungern, insbesondere während der Paarungszeit, vor der Häutung sowie bei Änderungen der Umweltbedingungen im Terrarium, Krankheit oder anderen externen Stressfaktoren (39). Das Terrarium dieses Pythons war jedoch sehr sorgfältig gestaltet und eingerichtet, sodass keine Risikofaktoren für Verletzungen oder die Anorexie

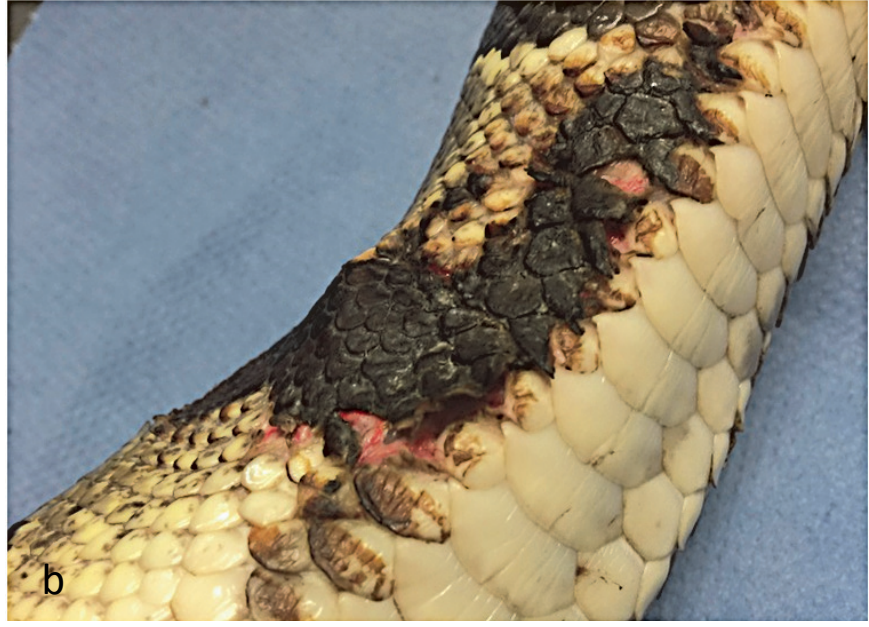

Fig. 1 Clinical presentation of the ball python. a) Sharply demarcated, necrotic skin area at the right flank in the middle of the body. b) Partially detaching eschar at the thorax revealing the underlying muscle layer.

erkennbar waren. Scharfkantige Objekte oder Hitzequellen, die für die Nekrosen und Hautwunden hätten verantwortlich sein können, wurden anamnestisch ausgeschlossen.

Bislang gibt es nur wenige pharmakokinetische Studien bei Reptilien und die Dosierung von Antibiotika erfolgt oft empirisch oder basierend auf anekdotischen Informationen (23). Trotz limitierter pharmakokinetischer Daten werden Fluorchinolone regelmäßig zur Behandlung bakterieller Infektionen bei Schlangen eingesetzt (40). Diese sind effektiv gegen die meisten bei Reptilien vorkommenden aeroben Bakterienstämme und verteilen sich gut

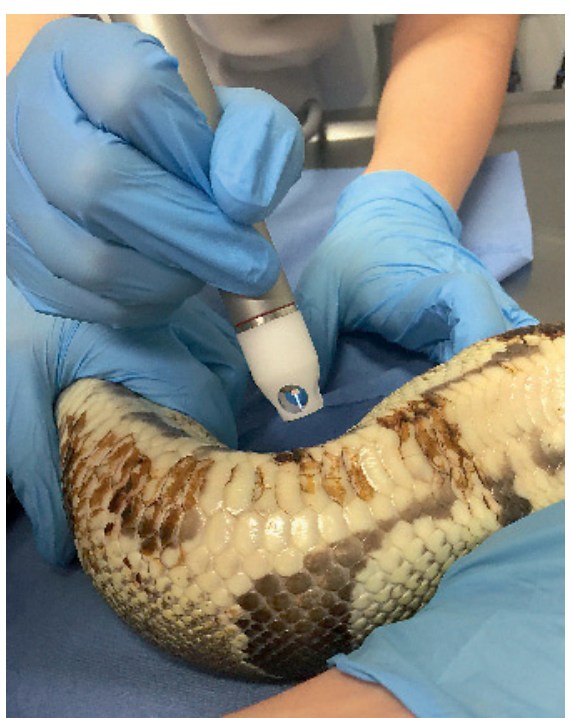

Abb. 2 Durchführung der Kaltplasmatherapie (nekrotisches Hautareal aufgrund des Fotografiewinkels nicht sichtbar). Jeder Quadratzentimeter wurde über 15 Sekunden behandelt.

Fig. 2 Process of cold plasma therapy (necrotic skin area not visible due to angle of photo). Each square centimeter was treated for 15 seconds. 


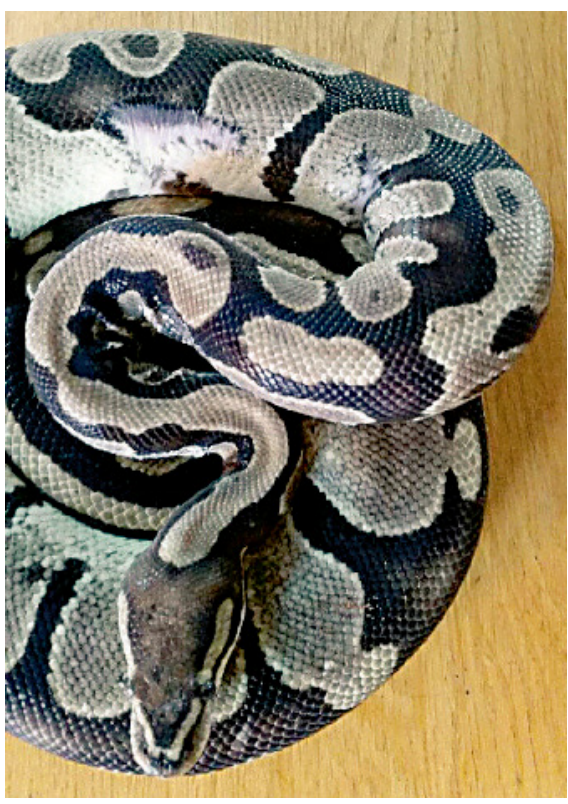

Abb. 3 Zustand des Patienten nach 4 Monaten. Obwohl Narbengewebe an den ehemaligen Wundstellen verblieb, zeigte die Schlange keine Anzeichen für Dysecdysis.

Fig. 3 Condition of the patient after 4 months. Despite scar tissue persisted on the former wounds, the snake has shown no symptoms of dysecdysis.

in alle Gewebe, einschließlich Knochen und Entzündungsgewebe (27). Nebenwirkungen auf Fluorchinolone treten bei Reptilien sehr selten auf, doch wurde von Schmerzreaktionen an der Injektionsstelle berichtet (25). Nahezu alle Isolate von Anaerobiern bei Schlangen erwiesen sich als sensitiv gegenüber Makrolidantibiotika (34).

Multiresistente Bakterien sind ein weltweit zunehmendes Problem (7) und zumindest teilweise auf den häufigen Antibiotikaeinsatz zurückzuführen (17). Die gängigen Antibiotika-Leitlinien empfehlen daher einen initialen Ansatz mit nur einem Antibiotikum $(5,13)$. Bei diesem Patienten mit Septikämie und neurologischen Ausfallerscheinungen erschienen jedoch aerobe wie auch anaerobe Bakterien als plausible Krankheitserreger. Da derzeit kein intramuskulär injizierbares Monopräparat für Reptilien in Deutschland zugelassen ist, das zur Behandlung beider Bakterientypen geeignet wäre, wurde bereits vor Vorliegen des Antibiogramms ein Ansatz mit zwei Antibiotika gewählt.

Stenotrophomonas (S.) maltophilia kann bei Patienten mit geschwächtem Immunsystem verheerende Infektionen wie auch eine

\section{Fazit für die Praxis}

Die Kaltplasmatherapie könnte eine nützliche Behandlungsoption für bakterielle Pyodermien und nekrotisierende Dermatitiden bei Schlangen darstellen, insbesondere in Fällen von multiresistenten bakteriellen Infektionen.
Septikämie bedingen (1). Der Erreger wurde als Ursache für schwere Infektionen bei Reptilien beschrieben (11), kann jedoch auch Teil der physiologischen Maulflora von Schlangen sein (12). Es bleibt daher ungeklärt, ob die bakterielle Infektion wirklich durch die Ratte oder durch ein Selbsttrauma des Pythons beim Kampf mit dem Beutetier verursacht wurde.

Ebenso ließ sich nicht klären, ob S. maltophilia auch die systemischen und neurologischen Symptome bedingte oder lediglich für die Hautulzerationen verantwortlich war, da der Besitzer vorgeschlagene Untersuchungen wie Blutkulturen, Blutbild, Serumbiochemie, tiefe bakterielle Kulturen oder eine histopathologische Untersuchung aus Kostengründen ablehnte. Trotz des deutlich verbesserten Allgemeinbefindens des Patienten verschlechterte sich der Zustand der Hautwunden zunehmend. Vermutet wurde daher eine beeinträchtigte Penetration der systemisch applizierten Antibiotika, z. B. durch die Gewebenekrose, und/oder eine bakterielle Resistenz auf die topischen Medikamente. Eine Pilzinfektion konnte nicht vollkommen ausgeschlossen werden, da aus Kostengründen auch eine Pilzkultur unterblieb. Im Fall einer solchen Infektion wäre allerdings eher eine histiozytäre Entzündung zu erwarten und bei der zytologischen Untersuchung wurden keinerlei Pilzelemente identifiziert. Ferner erscheint aufgrund des perakuten Krankheitsverlaufs in Kombination mit der heterophilen Entzündung und den zahlreichen zytologisch nachgewiesenen Bakterien eine bakterielle Infektion als wahrscheinlicher. Eine weitere Ursache für neurologische Symptome bei Riesenschlangen sind virale Infektionen. Der Python wurde jedoch über 3 Jahre einzeln gehalten und der zeitliche Zusammenhang zwischen Hautinfektion und Bissverletzungen macht eine bakterielle Pathogenese - insbesondere auch in Anbetracht des Therapieerfolgs - wahrscheinlicher. Zudem wurden bei der zytologischen Untersuchung von Proben aus den Hautwunden keine intrazytoplasmatischen oder intranukleären Einschlusskörperchen gefunden. Eine Untersuchung auf Virusantikörper und eine Liquoranalyse lehnte der Besitzer aus fianziellen Gründen ab.

Dank seiner wundheilungsfördernden und antimikrobiellen Effekte ist Kaltplasma eine neuartige, schmerzfreie und gut tolerierte Therapieoption für Wundbehandlungen $(3,37)$. Es handelt sich dabei um ein physikalisches Gemisch aus Elektronen und Ionen mit geladenen Molekülen in einem elektronisch erzeugten Gas $(9,20)$. Kaltplasma wirkt bakteriostatisch gegen Methicillin-resistenten Staphylococcus aureus (MRSA) und Pseudomonas aerugino$s a(2,28)$ und ist nachgewiesenermaßen effektiv gegen Virus- und Pilzinfektionen $(18,35)$ wie auch die Bildung von Biofilmen (2). Ferner beeinflusst es verschiedene Zellelemente wie Lipide, Proteine und die DNS durch seine geladenen und aktiven Moleküle (freie Radikale, Peroxide und andere reaktive Spezies) wie auch die Produktion und Sekretion von Wachstumsfaktoren $(32,38)$. Der baulich vergleichbare Plasma-Jet "kINPen Med" wird in der $\mathrm{Hu}$ manmedizin für die Behandlung von schlecht heilenden Wunden und Ulzerationen eingesetzt $(14,21)$ und besitzt antimikrobielle Aktivität bei Tieren und Menschen $(15,18)$. Die Eindringtiefe von Kaltplasma wurde mittels Mikrospektroskopie gemessen und be- 
trägt etwa $10 \mu \mathrm{m}$ in intakte menschliche Haut $(8,22)$. Schlangenhaut ist Studien zufolge etwa $14-40 \mu \mathrm{m}$ dick in mehreren überlappenden Schichten (19) und durch epidermale Schuppen und Hautfalten geprägt $(10,19)$. Bei unserem Patienten hat sehr wahrscheinlich die durch die Ulzerationen geschädigte Hautbarriere einen klinisch relevanten Effekt des Kaltplasmas ermöglicht. Es wurden keinerlei Nebenwirkungen der Behandlung festgestellt.

Nach bestem Wissen der Autoren ist dies der erste Fallbericht, der eine erfolgreiche Kaltplasmabehandlung einer multiresistenten bakteriellen Infektion bei einem Reptil beschreibt. Dieses Verfahren könnte somit eine vielversprechende Therapieoption für Infektionen bei diesen Spezies sein.

\section{Interessenkonflikt}

Die Autoren bestätigen, dass kein Interessenkonflikt besteht.

\section{Danksagung}

Die Autoren möchten der Firma neoplas tools $\mathrm{GmbH}$ (die nicht in die Patienten- oder Therapiewahl involviert war) für die Bereitstellung der Kaltplasmaeinheit an die Medizinische Kleintierklinik danken.

\section{Literatur}

1. Abbott IJ, Slavin MA, Turnidge JD, Thursky KA, Worth LJ. Stenotrophomonas maltophilia: emerging disease patterns and challenges for treatment. Expert Rev Anti Infect Ther 2011; 9: 471-488.

2. Alkawareek MY, Algwari QT, Laverty G, Gorman SP, Graham WG, O‘Connell D, Gilmore BF. Eradication of Pseudomonas aeruginosa biofilms by atmospheric pressure non-thermal plasma. PLoS One 2012; 7: e44289.

3. Arndt S, Unger P, Wacker E, Shimizu T, Heinlin J, Li YF, Thomas HM, Morfill GE, Zimmermann JL, Bosserhoff AK, Karrer S. Cold atmospheric plasma (CAP) changes gene expression of key molecules of the wound healing machinery and improves wound healing in vitro and in vivo. PLoS One 2013; 8: e79325.

4. Barten SL. Bites from prey. In: Reptile Medicine and Surgery, 2nd edn. Mader DR, ed. Saint Louis: Saunders 2006; 747-750.

5. Beco L, Guaguere E, Lorente Mendez C, Noli C, Nuttall T, Vroom M. Suggested guidelines for using systemic antimicrobials in bacterial skin infections: part 2 - antimicrobial choice, treatment regimens and compliance. Vet Rec 2013; 172: 156-160.

6. Cushing A, Pinborough M, Stanford M. Review of bacterial and fungal culture and sensitivity results from reptilian samples submitted to a UK laboratory. Vet Rec 2011; 169: 390.

7. Dennesen PJ, Bonten MJ, Weinstein RA. Multiresistant bacteria as a hospital epidemic problem. Ann Med 1998; 30: 176-185.

8. Fluhr JW, Sassning S, Lademann O, Darvin ME, Schanzer S, Kramer A, Richter H, Sterry W, Lademann J. In vivo skin treatment with tissue-tolerable plasma influences skin physiology and antioxidant profile in human stratum corneum. Exp Dermatol 2012; 21: 130-134.

9. Fridman A, Chirokov A, Gutsol A. Non-thermal atmospheric pressure discharges. J Phys D: Applied Physics 2005; 38: R1.

10. Funk RS. Snakes. In: Reptile Medicine and Surgery, 2nd edn. Mader DR, ed. Saint Louis: Saunders 2006; 42-58.

11. Harris NB, Rogers DG. Septicemia associated with Stenotrophomonas maltophilia in a West African dwarf crocodile (Osteolaemus tetraspis subsp. tetraspis). J Vet Diagn Invest 2001; 13: 255-258.

12. Hejnar P, Bardon J, Sauer P, Kolar M. Stenotrophomonas maltophilia as a part of normal oral bacterial flora in captive snakes and its susceptibility to antibiotics. Vet Microbiol 2007; 121: 357-362.
13. Hillier A, Lloyd DH, Weese JS, Blondeau JM, Boothe D, Breitschwerdt E, Guardabassi L, Papich MG, Rankin S, Turnidge JD, Sykes JE. Guidelines for the diagnosis and antimicrobial therapy of canine superficial bacterial folliculitis (Antimicrobial Guidelines Working Group of the International Society for Companion Animal Infectious Diseases). Vet Dermatol 2014; 25 : 163-175, e142-163.

14. Hubner NO, Matthes R, Koban I, Randler C, Muller G, Bender C, Kindel E, Kocher T, Kramer A. Efficacy of chlorhexidine, polihexanide and tissuetolerable plasma against Pseudomonas aeruginosa biofilms grown on polystyrene and silicone materials. Skin Pharmacol Physiol 2010; 23 Suppl: 28-34.

15. Koban I, Matthes R, Hübner NO, Welk A, Meisel P, Holtfreter B, Sietmann R, Kindel E, Weltmann KD, Kramer A, Kocher T. Treatment of Candida albicans biofilms with low-temperature plasma induced by dielectric barrier discharge and atmospheric pressure plasma jet. New J Phys 2010; 12: 073039 .

16. Kawarai T, Narisawa N, Yoneda S, Tsutsumi Y, Ishikawa J, Hoshino Y, Senpuku H. Inhibition of Streptococcus mutans biofilm formation using extracts from Assam tea compared to green tea. Arch Oral Biol; 68: 73-82.

17. Kerwat K, Kerwat M, Graf J, Wulf H. [Resistance to antibiotics and multiresistant pathogens]. Anasthesiologie, Intensivmedizin, Notfallmedizin, Schmerztherapie: AINS 2010; 45: 242-243.

18. Klampfl TG, Isbary G, Shimizu T, Li YF, Zimmermann JL, Stolz W, Schlegel J, Morfill GE, Schmidt HU. Cold atmospheric air plasma sterilization against spores and other microorganisms of clinical interest. Appl Environ Microbiol 2012; 78: 5077-5082.

19. Klein MC, Gorb SN. Epidermis architecture and material properties of the skin of four snake species. J R Soc Interface 2012; 9: 3140-3155.

20. Kogelschatz U. Atmospheric-pressure plasma technology. Plasma Phys Contr F 2004; 46: B63.

21. Kramer A, Lademann J, Bender C, Sckell A, Hartmann B, Münch S, Hinz P, Ekkernkamp A, Matthes R, Koban I, Partecke I, Heidecke CD, Masur K, Reuter S, Weltmann KD, Koch S, Assadian O. Suitability of tissue tolerable plasmas (TTP) for the management of chronic wounds. Clin Plasma Med 2013; 1: 11-18.

22. Lademann J, Ulrich C, Patzelt A, Richter H, Kluschke F, Klebes M, Lademann O, Kramer A, Weltmann KD, Lange-Asschenfeldt B. Risk assessment of the application of tissue-tolerable plasma on human skin. Clin Plasma Med 2013; 1: 5-10.

23. Lawrence K. Drug dosages for chelonians. Vet Rec 1984; 114: 150-151.

24. Maas AK, 3rd. Vesicular, ulcerative, and necrotic dermatitis of reptiles. Vet Clin North Am Exot Anim Pract 2013; 16: 737-755.

25. Mitchell MA. Therapeutics. In: Reptile Medicine and Surgery, 2nd edn. Mader DR, ed. Saint Louis: Saunders 2006; 2006; 631-664.

26. Montali RJ, Bush M, Smeller JM. The pathology of nephrotoxicity of gentamicin in snakes. A model for reptilian gout. Vet Pathol 1979; 16: 108-115.

27. Montay G, Goueffon Y, Roquet F. Absorption, distribution, metabolic fate, and elimination of pefloxacin mesylate in mice, rats, dogs, monkeys, and humans. Antimicrob Agents Chemother 1984; 25: 463-472.

28. Napp M, Daeschlein G, von Podewils S, Hinz P, Emmert S, Haase H, Spitzmueller R, Gumbel D, Kasch R, Junger M. In vitro susceptibility of methicillin-resistant and methicillin-susceptible strains of Staphylococcus aureus to two different cold atmospheric plasma sources. Infect 2016; 44: 531-537.

29. Gopee NV, Adesiyun AA, Caesar K. A longitudinal study of Escherichia coli strains isolated from captive mammals, birds, and reptiles in Trinidad. J Zoo Wildl Med 2000; 31: 353-360.

30. Ash RJ, Mauck B, Morgan M. Antibiotic resistance of gram-negative bacteria in rivers, United States. Emerg inf Dis 2002; 8: 713-716.

31. Santoro M, Hernandez G, Caballero M. Aerobic bacterial flora of nesting green turtles (Chelonia mydas) from Tortuguero National Park, Costa Rica. J Zoo Wildl Med 2006; 37: 549-552.

32. Schmidt A, Wende K, Bekeschus S, Bundscherer L, Barton A, Ottmuller K, Weltmann KD, Masur K. Non-thermal plasma treatment is associated with changes in transcriptome of human epithelial skin cells. Free Radic Res 2013; 47: 577-592. 
33. Smith DA, Barker IK, Allen OB. The effect of certain topical medications on healing of cutaneous wounds in the common garter snake (Thamnophis sirtalis). Can J Vet Res 1988; 52: 129-133.

34. Stewart JS. Anaerobic bacterial infections in reptiles. J Zoo Wildl Med 1990; $21: 180-184$

35. Trompeter FJ, Neff WJ, Franken O, Heise M, Neiger M, Shuhai L, Pietsch GJ, Saveljew AB. Reduction of Bacillus Subtilis and Aspergillus Niger spores using nonthermal atmospheric gas discharges. IEEE T Plasma Sci 2002; 30: 1416-1423.

36. Uetz P, Hošek J. The Reptile Database. 20 Aug 2016. http://www.reptiledatabase.org/.
37. von Woedtke T, Metelmann HR, Weltmann KD. Clinical plasma medicine: state and perspectives of in vivo application of cold atmospheric plasma. Contrib Plasma Phys 2014; 54: 104-117.

38. Wende K, Barton A, Bekeschus S, Bundscherer L, Schmidt A, Weltmann $\mathrm{KD}$, Masur K. Proteomic tools to characterize non-thermal plasma effects in eukaryotic cells. Plasma Med 2013; 3: 81-95.

39. Wilkinson SL. Reptile wellness management. Vet Clin North Am Exot Anim Pract 2015; 18: 281-304.

40. Young LA, Schumacher J, Papich MG, Jacobson ER. Disposition of enrofloxacin and its metabolite ciprofloxacin after intramuscular injection in juvenile Burmese pythons (Python molurus bivittatus). J Zoo Wildl Med 1997; 28: 71-79. 